\title{
The Iterative Solver Template Library
}

\author{
Markus Blatt and Peter Bastian \\ Interdisciplinary Centre for Scientific Computing (IWR), University Heidelberg, Im \\ Neuenheimer Feld 368, 69120 Heidelberg, Germany \\ Markus.Blatt@iwr.uni-heidelberg.de, Peter.Bastian@iwr.uni-heidelberg.de \\ http://www.dune-project.org
}

\begin{abstract}
The numerical solution of partial differential equations frequently requires the solution of large and sparse linear systems. Using generic programming techniques like in $\mathrm{C}++$ one can create solver libraries that allow efficient realization of "fine grained interfaces", i. e. with functions consisting only of a few lines, like access to individual matrix entries. This prevents code replication and allows programmers to work more efficiently.

In this paper we present the "Iterative Solver Template Library" (ISTL) which is part of the "Distributed and Unified Numerics Environment" (DUNE). It applies generic programming in $\mathrm{C}++$ to the domain of iterative solvers of linear systems stemming from finite element discretizations. Those discretizations exhibit a lot of structure. Our matrix and vector interface supports a block recursive structure. I. E. each sparse matrix entry can be a sparse or a small dense matrix itself. Based on this interface we present efficient solvers that use the recursive block structure via template metaprogramming.
\end{abstract}

\section{Introduction}

The numerical solution of partial differential equations (PDEs) frequently requires solving of large and sparse linear systems. Naturally, there are many libraries available for doing sparse matrix/vector computations, see [7] for a comprehensive list.

The widely availably Basic Linear Algebra Subprograms (BLAS) standard has been extended to cover also sparse matrices [5]. The standard uses procedural programming style and offers only a FORTRAN and C interface. "Fine grained" interfaces, meaning that functions consisting only of a few lines of code, such as access to individual matrix elements, are not slow compared to big functions, are not possible in this setup.

Generic programming techniqes in $\mathrm{C}++$ offer the possibility to combine flexibility and reuse ("efficiency of the programmer") with fast execution ("efficieny of the program"). This has been demonstrated with the Standard Template Library (STL), [15] or the Blitz++ library [6]. For an introduction to generic programming for scientific computing see $[2,16]$. Application of these ideas to matrix/vector operations is available with the Matrix Template Library (MTL), 
$[12,14]$ and to iterative solvers for linear systems with the Iterative Template Library (ITL), [11].

In contrast to these libraries the "Iterative Solver Template Library" (ISTL), which is part of the "Distributed and Unified Numerics Environment" (DUNE), $[3,8]$, is designed specifically for linear systems stemming from finite element discretizations. The sparse matrices representing these linear systems exhibit a lot of structure, e. g.:

- Certain discretizations for systems of PDEs or higher order methods result in matrices where individual entries are replaced by small blocks, say of size $2 \times 2$ or $4 \times 4$, see Fig. 1(a). Dense blocks of different sizes e. g. arise in $h p$ Discontinuous Galerkin discretization methods, see Fig. 1(b). Straightforward iterative methods solve these small blocks exactly, see e. g. [4].

- Equation-wise ordering for systems results in matrices having an $n \times n$ block structure where $n$ corresponds to the number of variables in the PDE and the blocks themselves are large. As an example we mention the Stokes system, see Fig. 1(d). Iterative solvers such as the SIMPLE or Uzawa algorithm use this structure.

- Other discretizations, e. g. those of reaction/diffusion systems, produce sparse matrices whose blocks are sparse matrices of small dense blocks, see 1(c).

- Other structures that can be exploited are the level structure arising from hierarchic meshes, a p-hierarchic structure (e. g. decomposition in linear and quadratic part), geometric structure from decomposition in subdomains or topological structure where unknowns are associated with nodes, edges, faces or elements of a mesh.

This structure is typically known at compile-time and therefore should be exploited to produce efficient code. Moreover, block structuredness is recursive, i. e. matrices are build from blocks which can themselves be build from blocks.

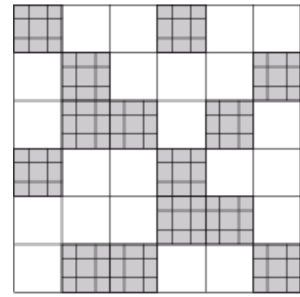

(a)

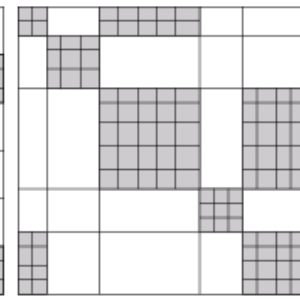

(b)

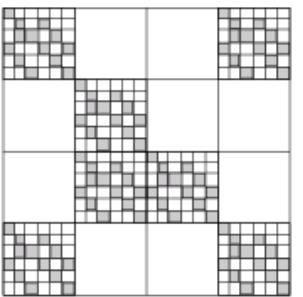

(c)

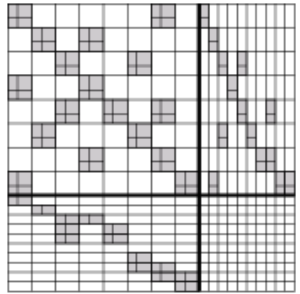

(d)

Fig. 1. Block structure of matrices arising in the finite element method

In the next section we describe the matrix and vector interface that represents this recursive block structure via templates. In Sect. 3 we show how to exploit the block structure using template metaprogramming at compile time. Finally we sketch the high level iterative solver interface in Sec. 4 . 


\section{Matrix and Vector Interface}

The interface of our matrices are designed according to what they represent from a mathematical point of view. The vector classes are representations of vector spaces while the matrix classes are representations of linear maps between two vector spaces.

\section{$2.1 \quad$ Vector Spaces}

We assume the reader is familiar with the concept of vector spaces. Essentially a vector space over a field $\mathbb{K}$ is a set $V$ of elements (called vectors) along with vector addition $+: V \mapsto V$ and scalar multiplication $\cdot: \mathbb{K} \times V \mapsto V$ with the well known properties. See your favourite textbook for details, e. g. [10].

For our application the following way of construction plays an important role: Let $V_{i}, i=1,2, \ldots, n$, be a normed vector spaces of dimension $n_{i}$ with a scalarproduct, then the $n$-nary cartesian product

$$
V:=V_{1} \times V_{2} \times \ldots \times V_{k}=\left\{\left(\boldsymbol{v}_{1}, \boldsymbol{v}_{2}, \ldots, \boldsymbol{v}_{n}\right) \mid \boldsymbol{v}_{1} \in V_{1}, \boldsymbol{v}_{2} \in V_{2}, \ldots, \boldsymbol{v}_{n} \in V_{n}\right\}
$$

is again a normed vector space of dimension $\sum_{i=1}^{n} n_{i}$ with the canonical norm and scalarproduct.

Treating $\mathbb{K}$ as a vector space itself we can apply this construction recursively starting from the field $\mathbb{K}$.

While for a mathematician every finite dimensional vector space is isomorphic to $\mathbb{R}^{k}$ for an appropriate $k$ for our application it is important to know how the vector space was constructed recursively by the procedure described in (1).

Vector Classes. To express the construnction of the vector space by $n$-nary products of other vector spaces ISTL provides the following classes:

FieldVector. The template $<$ class $\mathrm{K}$, int $\mathrm{n}>$ FieldVector $\langle\mathrm{K}, \mathrm{n}\rangle$ class template is used to represent a vector space $V=\mathbb{K}^{n}$ where the field is given by the type K. K may be double, float, complex<double> or any other numeric type. The dimension given by the template parameter $\mathrm{n}$ is assumed to be small.

Example: Use FieldVector<double,2> for vectors with a fixed dimension 2.

BlockVector. The template<class B $>\mathrm{BlockVector}<\mathrm{B}>$ class template builds a vector space $V=B^{n}$ where the "block type" $B$ is given by the template parameter B. B may be any other class implementing the vector interface. The number of blocks $n$ is given at run-time.

Example: BlockVector $<$ FieldVector $<$ double, $2>>$ can be used to define vectors of variable size where each block in turn consists of two double values. 
VariableBlockVector. The template $<$ class B $>$ VariableBlockVector $<\mathrm{B}>$ class can be used to construct a vector space having a two-level block structure of the form $V=B^{n_{1}} \times B^{n_{2}} \times \ldots \times B^{n_{m}}$, i.e. it consists of $m$ blocks $i=1, \ldots, m$ and each block in turn consists of $n_{i}$ blocks given by the type B. In principle this structure could be built also with the previous classes but the implementation here is more efficient. It allocates memory in one big array for all components. For certain operations it is more efficient to interpret the vector space as $V=B^{N}$, where $N=\sum_{i=1}^{m} n_{i}$.

Vectors are containers. Vectors are containers over the base type $\mathrm{K}$ or $\mathrm{B}$ in the sense of the Standard Template Library. Random access is provided via operator [] (int i) where the indices are in the range $0, \ldots, n-1$ with the number of blocks $n$ given by the $\mathrm{N}$ method. Here is a code fragment for illustration:

typedef Dune: :FieldVector<std: : complex<double>,2> BType;

Dune: : BlockVector $\langle$ BType $>\mathrm{v}(20)$;

$\mathrm{v}[1]=3.14$

$\mathrm{v}[3][0]=2.56$;

$\mathrm{v}[3][1]=\operatorname{std}:$ : complex $\langle$ double $>(1,-1)$;

Note how one operator [] () is used for each level of block recursion.

Sequential access to container elements is provided via iterators. The Iterator class provides read/write access while the ConstIterator provides read-only access. The type names are accessed via the ::-operator from the scope of the vector class.

A uniform naming scheme enables writing of generic algorithms. See Table 1 for the types provided in the scope of any vector class.

Table 1. Types of vector classes

\begin{tabular}{|c|c|}
\hline expression & return type \\
\hline field_type & $\begin{array}{l}\text { The type of the field of the repesented vector space, e. g. } \\
\text { double. }\end{array}$ \\
\hline block_type & The type of the blocks vector. \\
\hline size_type & The type used for the index access and size operations. \\
\hline block_level & $\begin{array}{l}\text { The block level of the vector, e. g. } 1 \text { for FieldVector, } 2 \text { for } \\
\text { BlockVector }\langle\text { FieldVector }\langle K\rangle, n\rangle \text {. }\end{array}$ \\
\hline Iterator & The type of the iterator. \\
\hline ConstIterator & The type of the immutable iterator. \\
\hline
\end{tabular}

\subsection{Linear maps}

For a matrix representing a linear map (or homomorphism) $A: V \mapsto W$ from vector space $V$ to vector space $W$ the recursive block structure of the matrix rows and columns immediatly follows from the recursive block structure of the 
vectors representing the domain and range of the mapping, respectively. As a natural consequence we designed the following matrix classes:

Matrix classes. Using the construction in (1) the structure of our vector spaces carries over to linear maps in a natural way.

FieldMatrix. the template $<$ class $\mathrm{K}$, int $\mathrm{n}>$ FieldMatrix $<\mathrm{K}, \mathrm{n}, \mathrm{m}>$ class template is used to represent a linear map $M: V_{1} \rightarrow V_{2}$ where $V_{1}=\mathbb{K}^{n}$ and $V_{2}=\mathbb{K}^{m}$ are vector spaces over the field given by template parameter $\mathrm{K}$. K may be double, float, complex<double $>$ or any other numeric type. The dimensions of the two vector spaces given by the template parameters $\mathrm{n}$ and $\mathrm{m}$ are assumed to be small. The matrix is stored as a dense matrix. Example: Use FieldMatrix<double , 2,3> to define a linear map from a vector space over doubles with dimension 2 to one with dimension 3 .

BCRSMatrix. The template<class B> BCRSMatrix class template represents a sparse matrix where the "block type" $B$ is given by the template parameter B. B may be any other class implementing the matrix interface. The matrix class uses a compressed row storage scheme.

VariableBCRSMatrix. The template<class B> VariableBCRSMatrix<B> class can be used to construct a linear map between two vector spaces having a two-level block structure $V=B^{n_{1}} \times B^{n_{2}} \times \ldots \times B^{n_{m}}$ and $W=B^{m_{1}} \times B^{m_{2}} \times \ldots \times B^{m_{k}}$. Both are represented by the template $\langle$ class $B>$ VariableBlockVector $\langle\mathrm{B}\rangle$ class, see 2.1. This is not implemented yet.

Matrices are containers of containers. Matrices are containers over the matrix rows. The matrix rows are containers over the type $\mathrm{K}$ or $\mathrm{B}$ in the sense of the Standard Template Library. Random access is provided via operator [] (int i) on the matrix to the matrix rows and on the matrix rows to the matrix columns (if present). Note that except for FieldMatrix, which is a dense matrix, operator [] on the matrix row triggers a binary search for the column.

For sequential access use RowIterator and ColIterator for read/write access or ConstRowIterator and ConstColIterator for readonly access to rows and columns, respectively. Here is a small example that prints the sparsity pattern of a matrix of type M:

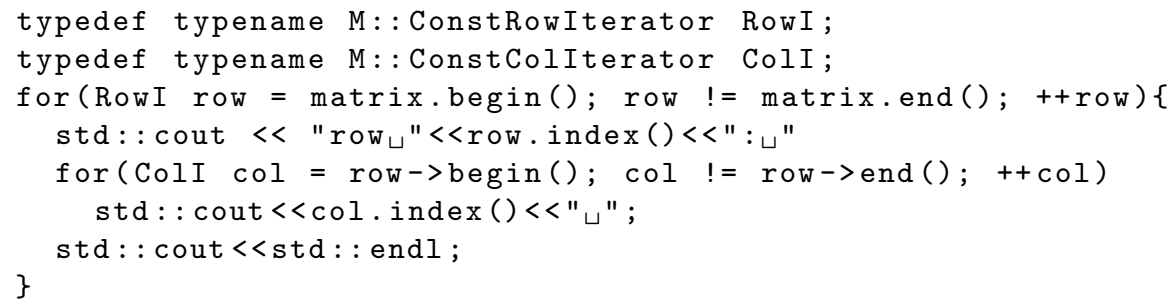

As with the vector interface a uniform naming convention enables generic algorithms. See Table 2 for the most important names. 
Table 2. Type names in the matrix classes

\begin{tabular}{|c|c|}
\hline expression & return type \\
\hline field_type & $\begin{array}{l}\text { The type of the field of the vector spaces we map from and } \\
\text { to }\end{array}$ \\
\hline block_type & Th type representing the matrix components \\
\hline row_type & The container type of the rows. \\
\hline size_type & The type used for index access and size operations \\
\hline block_level & $\begin{array}{l}\text { The block recursion level, e. g. } 1 \text { for FieldMatrix and } 2 \text { for } \\
\text { BlockVector }\langle\text { FieldVector }\langle\mathrm{K}\rangle, \mathrm{m}, \mathrm{n}\rangle \text {. }\end{array}$ \\
\hline RowIterator & The type of the mutable iterator over the rows \\
\hline ConstRowIterator & dito, but immutable \\
\hline $\begin{array}{l}\text { ColIterator } \\
\text { ConstColIterator }\end{array}$ & $\begin{array}{l}\text { The type of the mutable iterator over columns of a row. } \\
\text { dito, but immutable }\end{array}$ \\
\hline
\end{tabular}

\section{Block Recursive Algorithms}

\section{$3.1 \quad$ Block Recursion}

The basic feature of the concept described by the matrix and vector classes, is their recursive block structure. Let $A$ be a matrix with blocklevel $l>1$ then each block $A_{i j}$ can be treated as (or actually is) a matrix itself. This recursiveness can be exploited in generic algorithm using the defined block_level of the matrix and vector classes.

Most preconditioner can be modified to honor this recursive structure for a specific number of block levels $k$. They then work as normal on the offdiagonal blocks, treating them as traditional matrix entries. For the diagonal values a special procedure applies: If $k>1$ the diagonal is treated as a matrix itself and the preconditioner is applied recursively on the matrix representing the diagonal value $D=A_{i i}$ with blocklevel $k-1$. For the case that $k=1$ the diagonal is treated as a matrix entry resulting in a linear solve or an identity operation depending on the algorithm.

\subsection{Iterative Solver Kernels}

In the formulation of most iterative methods upper and lower triangular and diagonal solves play an important role. ISTL provides block recursive versions of these generic building blocks using template metaprogramming, see Table 3 for a listing of these methods. In the table matrix $A$ is decomposed into $A=L+D+U$, where $L$ is a strictly lower block triangular, $D$ is a block diagonal and $U$ is a strictly upper block triangular matrix. An arbitrary block recursion level can be given by an additional parameter. If this parameter is omitted it defaults to 1 .

Using the same block recursive template metaprogramming technique, kernels for the defect formulations of simple iterative solvers are available in ISTL. The number of block recursion levels can again be given as an additional argument. See the second part of Table 3 for a list of these kernels. 
Table 3. Iterative Solver Kernels

\begin{tabular}{|c|c|}
\hline function & computation \\
\hline \multicolumn{2}{|c|}{ block triangular and block diagonal solves } \\
\hline bltsolve $(A, v, d)$ & $v=(L+D)^{-1} d$ \\
\hline bltsolve $(A, v, d, \omega)$ & $v=\omega(L+D)^{-1} d$ \\
\hline ubltsolve $(A, v, d)$ & $v=L^{-1} d$ \\
\hline ubltsolve $(A, v, d, \omega)$ & $v=\omega L^{-1} d$ \\
\hline butsolve $(A, v, d)$ & $v=(D+U)^{-1} d$ \\
\hline butsolve $(A, v, d, \omega)$ & $v=\omega(D+U)^{-1} d$ \\
\hline ubutsolve $(A, v, d)$ & $v=U^{-1} d$ \\
\hline ubutsolve $(A, v, d, \omega)$ & $v=\omega U^{-1} d$ \\
\hline bdsolve $(A, v, d)$ & $v=D^{-1} d$ \\
\hline bdsolve $(A, v, d, \omega)$ & $v=\omega D^{-1} d$ \\
\hline \multicolumn{2}{|r|}{ iterative solves } \\
\hline $\operatorname{dbjac}(A, x, b, \omega)$ & \multirow{2}{*}{$\begin{array}{l}x=x+\omega D^{-1}(b-A x) \\
x=x+\omega(L+D)^{-1}(b-A x)\end{array}$} \\
\hline $\operatorname{dbgs}(A, x, b, \omega)$ & \\
\hline $\operatorname{bsorf}(A, x, b, \omega)$ & $x_{i}^{k+1}=x_{i}^{k}+\omega A_{i i}^{-1} \quad b_{i}-\sum_{j<i} A_{i j} x_{j}^{k+1}-\sum_{j \geq i} A_{i j} x_{j}^{k}$ \\
\hline $\operatorname{bsorb}(A, x, b, \omega)$ & $x_{i}^{k+1}=x_{i}^{k}+\omega A_{i i}^{-1} \quad b_{i}-\sum_{j \leq i} A_{i j} x_{j}^{k}-\sum_{j>i} A_{i j} x_{j}^{k+1}$ \\
\hline
\end{tabular}

\section{Solver Interface}

The solvers in ISTL do not work on matrices directly. Instead we use an abstract Operator concept. Thus we can even model and solve linear maps that are not stored as matrices (e. g. on the fly computed linear operators).

\subsection{Operators}

The base class template<class X, class $Y>$ LinearOperator represents linear maps. The template parameter $\mathrm{X}$ is the type of the domain and $\mathrm{Y}$ is the type of the range of the operator. A linear operator provides the methods apply (const $\mathrm{X} \& \mathrm{x}$ , Y\& y) and apply applyscaledadd (const $\mathrm{X} \& \mathrm{x}, \mathrm{Y} \& \mathrm{y}$ ) performing the operations $y=A(x)$ and $y=y+\alpha A(x)$, respectively. The subclass template<class $\mathrm{M}$, class $\mathrm{X}$, class $\mathrm{Y}>$ AssembledLinearOperator represents linear operators that have a matrix representation. Convertion from any matrix into a linear operator is done by the class template<class M, class X, class Y> MatrixAdapter.

\subsection{Scalarproducts}

For convergence tests and the stopping criteria Krylow methods need to compute scalar products and norms on the underlying vector spaces. The base class template<class X> Scalarproduct provides methods field_type dot(const X\& $\mathrm{x}$, const $\mathrm{x} \& \mathrm{y}$ ) and double norm (const $\mathrm{x} \& \mathrm{x}$ ) to calculate these. For sequential programs use template<class $\mathrm{X}>$ SeqScalarProduct which simply maps this to functions of the vector implementations. 


\subsection{Preconditioners}

The template $<$ class $\mathrm{X}$, class $\mathrm{Y}>$ Preconditioner provides the abstract base class for all precondioners in ISTL. The method void pre (X\& $\mathrm{x}, \mathrm{Y} \& \mathrm{~b}$ ) has to be called before applying the preconditioner. Here $\mathrm{x}$ is the left hand side and $\mathrm{b}$ is the right hand side of the operator equation. The method may, e. g. scale the system, allocate memory or compute an (I)LU decomposition. The method void apply ( $\mathrm{X} \& \mathrm{v}$, const $\mathrm{Y} \&$ ) applies one step of the preconditioner to the system $A(\boldsymbol{v})=\boldsymbol{d}$. Here $\mathrm{b}$ should contain the current defect and $\mathrm{v}$ should be 0 . Upon exit of the method v contains the computed update to the current guess, i. e. $\boldsymbol{v}=M^{-1} \boldsymbol{d}$ where $M$ is the approximate inverse of the operator $A$ characterizing the preconditioner. The method void post $(\mathrm{X} \& \mathrm{x}$ ) should be called after all computations to give the precondtioner the chance to clean allocated resources.

See Table 4 for a list of available preconditioner. They have the template

Table 4. Preconditioners

\begin{tabular}{|l|l|c|c|}
\hline class & implements & $\mathrm{s} / \mathrm{p}$ & recursive \\
\hline \hline SeqJac & Jacobi method & $\mathrm{s}$ & $\mathrm{x}$ \\
SeqSOR & successive overrelaxation (SOR) & $\mathrm{s}$ & $\mathrm{x}$ \\
SeqSSOR & Symmetric SSOR & $\mathrm{s}$ & $\mathrm{x}$ \\
SeqILU & incomplete LU decomposition (ILU) & $\mathrm{s}$ & \\
SeqILUN & ILU decpmposition of order N & $\mathrm{s}$ & \\
Pamg: : AMG & algebraic multigrid method & $\mathrm{s} / \mathrm{p}$ & \\
BlockPreconditioner & Additive overlapping Schwarz & $\mathrm{p}$ & \\
\hline
\end{tabular}

parameters $\mathrm{M}$ representing the type of the matrix they work on, $\mathrm{X}$ representing the type of the domain, $\mathrm{Y}$ representing the type of the range of the linear system. The block recursive preconditioner are marked with " $\mathrm{x}$ " in the last column. For them the recursion depth is specified via an additional template parameter int 1. The column labeled "s/p" specifies whether they support sequential and/or parallel mode.

\subsection{Solvers}

All solvers are subclasses of the abstract base class template<class X, class $Y>$ InverseOperator representing the inverse of an operator from the domain of type $\mathrm{X}$ to the range of type $\mathrm{Y}$. The actual solve of the system $A(\boldsymbol{x})=\boldsymbol{b}$ is done in the method void apply (X\& $x, Y \& b$, InverseOperatorResult\& $r$ ). In the Inverse0peratorResult some statistics about the solution process, e. g. iteration count, achieved defect reduction, etc., are stored. All solvers only use methods of instances of LinearOperator, ScalarProduct and Preconditioner. These are provided in the constructor.

See Table 5 for a list of available solvers. All solvers are template classes with a template parameter $\mathrm{x}$ providing them with the vector implementation used. 
Table 5. ISTL Solvers

\begin{tabular}{|l|l|}
\hline class & implements \\
\hline \hline LoopSolver & only apply precoditioner multiple time \\
GradientSolver & preconditioned radient method \\
CGSolver & preconditioned conjugate gradient method \\
BiCGStab & preconditioned biconjugate gradient stabilized method \\
\hline
\end{tabular}

\subsection{Parallel Solvers}

Instead of using parallel data structures (matrices and vectors) that (implicitly) know the data distribution and communication patterns like in PETSc $[13,1]$ we decided to decouple the parallelization from the data structures used. Basically we provide an abstract consistency model on top of our linear algebra. This is hidden in the parallel implementations of the interfaces of LinearOperator , Scalarproduct and Preconditioner, which assure consistency of the data (by communication) for the InverseOperator implementation. Therefore the same Krylow method algorithms work in parallel and sequential mode.

Based on the idea proposed in [9] we implemented parallel overlapping Schwarz preconditioners with inexact (sequential) subdomain solvers and a parallel algebraic multigrid preconditioner together with appropriate implementations of LinearOperator and Scalarproduct. Nonoverlapping versions are currently being worked on.

Note that using this approach it easy two switch form the currently implemented MPI version to new parallel programming paradigms that might be needed on new platforms.

\subsection{Performance Evaluation}

We evaluated the performance of our implementation on a Petium 4 Mobile 2.4 $\mathrm{GHz}$ with a measured memory bandwith of $1084 \mathrm{MB} / \mathrm{s}$ for the daypy operation $(x=y+\alpha z)$ in Tables 6 . The code was comiled with the GNU $\mathrm{C}++$ compiler

Table 6. Performance Tests

(a) scalar product

\begin{tabular}{|c|c|c|c|c|}
\hline 500 & 05000 & 50000 & 5000005 & 5000000 \\
\hline MFLOPS 896 & 775 & 167 & 160 & 164 \\
\hline
\end{tabular}

(c) Matrix-vector product, 5-point stencil, $b$ : block size

\begin{tabular}{|c|c|c|c|c|c|c|}
\hline$N, b$ & $100,110000,1$ & 1000000,1 & 1000000,2 & 1000000,3 & & C ISTL \\
\hline$\overline{M F L O P S}$ & 388 & 136 & 230 & 260 & time / it. $[\mathrm{s}$ & $\begin{array}{ll}0.17 & 0.18\end{array}$ \\
\hline
\end{tabular}

(b) daxpy operation $y=y+\alpha x$

$\overline{\overline{5005000500005000005000000}}$

\begin{tabular}{lllll}
\hline 936 & 910 & 108 & 103 & 107
\end{tabular}

(d) Damped Gauß-

Seidel

version 4.0 with -O3 optimization. In the tables $N$ is the number of unknown 
blocks (equals the number of unknows for the scalar cases in Tables 6(a), 6(b), $6(\mathrm{~d})$ ). The performance for the scalarproduct, see Table $6(\mathrm{a})$, and the daxpy operation, see Table 6(b) is nearly optimal and for large $N$ the limiting factor is clearly the memory bandwith. Table 6 (c) shows that we take advantage of cache reusage for matrices of dense blocks with block size $b>1$. In Table 6(d) we compared the generic implementation of the Gauss Seidel solver in ISTL with a specialized $\mathrm{C}$ implementation. The measured times per iteration show that there is now lack of computational efficiency due to the generic implementation.

\section{References}

1. S. Balay, K. Buschelman, V. Eijkhout, W. D. Gropp, D. Kaushik, M. G. Knepley, L. C. McInnes, B. F. Smith, and H Zhang. PETSc users manual. Technical Report ANL-95/11 - Revision 2.1.5, Argonne National Laboratory, 2004.

2. J. J. Barton and L. R. Nackman. Scientific and Engineering $C++$. Addison-Wesley, 1994.

3. P. Bastian, M. Droske, C. Engwer, R. Klöfkorn, T. Neubauer, M. Ohlberger, and M. Rumpf. Towards a unified framework for scientific computing. In R. Kornhuber, R. Hoppe, J. Priaux, O. Widlund O. Pironneau, and J. Xu, editors, Domain Decomposition Methods in Science and Engineering, volume 40 of LNCSE, pages 167-174. Springer-Verlag, 2005.

4. P. Bastian and R. Helmig. Efficient fully-coupled solution techniques for two-phase flow in porous media. Parallel multigrid solution and large scale computations. Adv. Water Res., 23:199-216, 1999.

5. BLAST Forum. Basic linear algebra subprograms technical (BLAST) forum standard, 2001. http://www.netlib.org/blas/blast-forum/.

6. Blitz++. http://www.oonumerics.org/blitz/.

7. J. Dongarra. List of freely available software for linear algebra on the web, 2006. http://netlib.org/utk/people/JackDongarra/la-sw.html.

8. DUNE. http://www. dune-project.org/.

9. G. Haase, U. Langer, and A. Meyer. The approximate dirichlet domain decomposition method. part i: An algebraic approach. Computing, 47:137-151, 1991.

10. J. Hefferson. Linear algebra, May 2006. http://joshua.amcvt.edu/.

11. Iterative template library. http://www.osl.iu.edu/research/itl/.

12. Matrix template library. http://www.osl.iu.edu/research/mtl/.

13. PETSc. http://www.mcs.anl.gov/petsc/.

14. J. Siek and A. Lumsdaine. A modern framework for portable high-performance numerical linear algebra. In H. P. Langtangen, A. M. Bruaset, and E. Quak, editors, Advances in Software Tools for Scientific Computing, volume 10 of LNCSE, pages 1-56. Springer-Verlag, 2000.

15. B. Stroustrup. The $C++$ Programming Language. Addison-Wesley, 1997.

16. T. Veldhuizen. Techniques for scientific $\mathrm{C}++$. Technical report, Indiana University, 1999. Computer Science Department. 